\title{
Conocimiento y conciencia de los médicos sobre la osteonecrosis relacionada con bifosfonatos.
}

\author{
Knowledge and awareness of physicians about \\ bisphosphonate-related osteonecrosis of the jaws.
}

\author{
Ilan Vinitzky-Brener, ${ }^{* \ddagger}$ Norma Guadalupe Ibáñez-Mancera,§ Ana Pilar Álvarez Jardón, ${ }^{\circledR}$ Lesly Johanna Serra-Rico," \\ Carlos Alberto Carrasco Rueda,* Rosario Fernández Plata,** David Martínez Briseño**
}

\section{RESUMEN}

Objetivo: Determinar el conocimiento y la conciencia que tienen los médicos sobre la osteonecrosis relacionada con bifosfonatos. Material y métodos: Se realizó un estudio transversal, en el cual se encuestó a médicos generales y especialistas con la finalidad de identificar el nivel de conocimientos y conciencia que tienen sobre el tema. Resultados: Se entrevistó a un total de 475 médicos generales y especialistas, de los cuales 210 (44.2\%) mencionaron prescribir bifosfonatos, de este grupo $58.1 \%$ no envía a los pacientes con el odontólogo para eliminar factores de riesgo, a pesar de que $61.8 \%$ de los mismos mencionó conocer las reacciones adversas; 36 médicos (17.4\%) han visto a algún paciente con osteonecrosis por bifosfonatos. El 37\% de los médicos que prescriben medicamentos consideran que no es necesario remitir a los pacientes al odontólogo. Conclusiones: La prescripción de bifosfonatos en la práctica médica va en aumento, los médicos deben tener el conocimiento adecuado sobre las reacciones adversas de estos medicamentos para así poder referir oportunamente al odontólogo, educar al paciente y poder prevenir complicaciones como la osteonecrosis relacionada con bifosfonatos.

Palabras clave: Conocimiento, conciencia, médicos, osteonecrosis, bifosfonatos.

\section{ABSTRACT}

Objectives : To evaluate the knowledge and awareness of physicians about bisphosphonate-related osteonecrosis of the jaws. Material and methods: A cross-sectional survey was carried out among general practitioners and specialized physicians to determine their knowledge and awareness of bisphosphonate-related osteonecrosis of the jaws. Results: Of the 475 interviewed general practitioners and specialized physicians, 210 (44.2\%) claimed to prescribe bisphosphonates. A total of $58.1 \%$ of these physicians did not refer their patients to the dentist for the elimination of risk factors, despite the fact that $61.8 \%$ of them reported knowledge of the adverse reactions of these drugs. Thirty-six physicians (17.4\%) had seen some patient with bisphosphonate-related osteonecrosis of the jaws. A total of $37 \%$ of the physicians that prescribed drugs considered it not necessary to refer patients to the dentist. Conclusions: Bisphosphonate prescription is increasingly common in medical practice, and physicians must have adequate knowledge of the adverse reactions of these drugs in order to ensure opportune patient referral to the dentist, educate their patients, and avoid complications such as bisphosphonate-related osteonecrosis of the jaws.

Keywords: Knowledge, awareness, physician, osteonecrosis, bisphosphonates.

\footnotetext{
* Departamento de Cirugía Maxilofacial del Instituto Nacional de Enfermedades Respiratorias Ismael Cosío Villegas. Ciudad de México, México.

‡ Facultad de Ciencias de la Salud de la Universidad Anáhuac México. Ciudad de México, México.

§ Centro Interdisciplinario de Ciencias de la Salud Unidad Santo Tomás (CICS-UST), Instituto Politécnico Nacional. Universidad Anáhuac México. Ciudad de México, México. ORCID: 0000-0002-3635-4850.

ף Universidad Anáhuac México. Ciudad de México, México. ORCID: 0000-0001-8721-2222.

| Universidad Latinoamericana. Ciudad de México, México. ORCID: 0000-0002-0267-7918.

** Departamento de Epidemiología del Instituto Nacional de Enfermedades Respiratorias Ismael Cosío Villegas. Ciudad de México, México.

Recibido: 23 de noviembre de 2020. Aceptado: 26 de enero de 2021.

Citar como: Vinitzky-Brener I, Ibáñez-Mancera NG, Álvarez JAP, Serra-Rico LJ, Carrasco RCA, Fernández PR et al. Conocimiento y conciencia de los médicos sobre la osteonecrosis relacionada con bifosfonatos. Rev ADM. 2021; 78 (1): 28-32. https://dx.doi.org/10.35366/98384
}

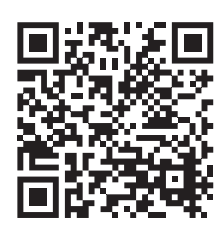




\section{INTRODUCCIÓN}

L os bifosfonatos son un grupo de medicamentos utilizados para la prevención y el tratamiento de diversas patologías que afectan a los huesos, entre los cuales destacan la osteoporosis/osteopenia, mieloma múltiple, metástasis ósea, hipercalcemia, enfermedad de Paget, entre otras. ${ }^{1}$ Su uso se ha popularizado en las décadas pasadas entre múltiples especialidades médicas con resultados favorables. ${ }^{2,3}$ Sin embargo, en 2003, R. Marx realizó el primer reporte en la literatura médica que relaciona el consumo de bifosfonatos con exposición ósea en los maxilares; ${ }^{4}$ posteriormente, en 2004, Ruggiero publicó una serie de 63 pacientes que presentaron osteonecrosis asociada con dichos medicamentos. ${ }^{5}$ A partir de estas publicaciones la comunidad científica ha estudiado esta reacción adversa y la cantidad de publicaciones sobre el tema es considerable. En 2007 la Asociación Americana de Cirugía Oral y Maxilofacial (American Association of Oral and Maxillofacial Surgeons [AAOMS]) publicó el primer «position paper» del tema, ${ }^{6}$ después en $2009^{7}$ se publicó una actualización y en 2014 un nuevo artículo, ahora utilizando el término «medication-related osteonecrosis of the jaw $\rangle^{8}$ para referirse a la misma condición.

La osteonecrosis de los maxilares relacionada con bifosfonatos (OMRB) es una patología caracterizada por la presencia de hueso expuesto en la región maxilofacial por al menos ocho semanas en un paciente que recibe o ha recibido terapia con bifosfonatos y que no ha estado expuesto a radiación en cabeza y cuello. ${ }^{7}$ Resulta complicado evaluar la incidencia verdadera de la OMRB ${ }^{9}$ por la variedad de esquemas terapéuticos y factores relacionados. Una revisión sistemática de la literatura publicada de manera reciente ha estimado la incidencia en pacientes oncológicos en un rango de 0 a 12.2 por cada 100,000 pacientes por año. ${ }^{10} \mathrm{Si}$ bien es cierto que la incidencia es baja, cuando se presenta la enfermedad el tratamiento es complejo y en ocasiones puede ser mutilante, lo que conlleva a una afectación importante en la calidad de vida de los pacientes. Como en la mayoría de las enfermedades, uno de los aspectos claves es la prevención, en el caso de la OMRB es indispensable que tanto el odontólogo como el médico tengan un conocimiento adecuado sobre el tema, de tal forma que el odontólogo al recibir a un paciente que se encuentra o ha estado bajo un tratamiento con bifosfonatos deba saber las pautas de tratamiento del mismo y el médico que prescribe bifosfonatos informe a los pacientes del riesgo de desarrollar osteonecrosis y envíe al paciente a un tratamiento odontológico previo. ${ }^{1,8}$
En el caso de los odontólogos las publicaciones han sido poco alentadoras, en 2010 Yoo y colaboradores publicaron un estudio sobre el conocimiento de los odontólogos en Corea sobre el tema, encontraron que únicamente $31.4 \%$ de los encuestados registran en la historia clínica antecedentes relacionados con estos medicamentos y mencionan que la mayoría de los encuestados no está al tanto de las guías publicadas por la AAOMS. ${ }^{11}$ Alhussain y colegas, en 2015, encuestaron a los odontólogos que practican en Ontario, Canadá, y reportaron que 50\% de los encuestados respondió no sentirse cómodo tratando pacientes que consumen bifosfonatos y sólo $23 \%$ sigue las guías publicadas para el tratamiento quirúrgico. ${ }^{12}$ En un estudio realizado por nuestro grupo en el que se encuestó a más de 400 odontólogos mexicanos encontramos que $99.3 \%$ de los participantes no posee un conocimiento suficiente para el diagnóstico y manejo de un paciente que recibe bifosfonatos..$^{13}$ Existen pocos estudios publicados que analicen el conocimiento $y$ la conciencia que tienen los médicos sobre el tema, y ya que ellos son los que prescriben los bifosfonatos resulta importante conocer estos datos. El presente estudio tiene por objeto conocer el nivel de conocimientos y la conciencia que tienen los médicos generales y especialistas en México sobre la OMRB, para así poder implementar las estrategias necesarias que permitan disminuir el número de casos que se presentan con esta patología.

\section{MATERIAL Y MÉTODOS}

Se realizó un estudio transversal, utilizando una encuesta con la finalidad de conocer el conocimiento y la conciencia de los médicos en México para la prevención de la OMRB. Se realizó un muestreo probabilístico, quedando una muestra de 475 médicos tanto de práctica general como especialistas, sin importar si su práctica profesional era privada, institucional o ambas. El cuestionario estuvo organizado en dos bloques: el primero con ítems sobre los datos generales de los médicos: especialidad (en caso de tenerla), tipo de práctica, tiempo de ejercer y aspectos generales de los bifosfonatos; el segundo bloque sólo se aplicó a los médicos que prescriben medicamentos de este grupo, se les interrogó sobre sus indicaciones, el término osteonecrosis por bifosfonatos, así como los riesgos y prevención de la misma. Las respuestas fueron valoradas por expertos basados en la literatura más reciente sobre el tema.

\section{RESULTADOS}

Se entrevistó a un total de 475 médicos, de los cuales 185 (38.9\%) eran médicos generales, 183 (38.5\%) especialis- 
tas y 107 (22.5\%) contaban con maestría y/o doctorado. La especialidad más común entre los entrevistados fue Ginecología y Obstetricia en 14\%, seguido por Pediatría en $4.8 \%$, después Medicina Interna con $4.2 \%$; además, dentro de la muestra se entrevistaron especialistas en Otorrinolaringología, Oftalmología, Oncología, Cirugía, Urología, Urgencias, etcétera. Respecto al tiempo que llevan ejerciendo la medicina, más de tres cuartas partes refirieron menos de cinco años (372 médicos, 78.3\%), 9.5\% (45 participantes) de cinco a 15 años y $12.2 \%$ más de 15 años de ejercicio profesional. Con respecto a su afiliación a algún consejo o asociación médica la distribución fue prácticamente igual, 50.1\% no pertenecen y $49.9 \%$ sí pertenecen a alguna instancia de este tipo. El 85.9\% de los médicos entrevistados mencionó que tomó al menos un curso de actualización al año. Poco más de la tercera parte de la muestra (36.6\%) además de su práctica como médicos, también realizan labores de docencia.

Se interrogó a los participantes sobre sus conocimientos sobre bifosfonatos, 61.5\% (292 médicos) contestaron que sí los conocen. Posteriormente, para verificar el conocimiento, se les pidió mencionar la sustancia activa de dos medicamentos de este grupo, se obtuvo que la mitad de los entrevistados (239) no respondieron ninguno, $21 \%$ sólo respondieron un medicamento y el restante $29 \%$ sí respondieron los nombres de dos medicamentos. Con respecto al nombre comercial, 60\% (285 médicos) no contestaron ninguno, $20 \%$ respondieron uno y $20 \%$ respondieron los dos nombres.

De los 475 médicos entrevistados, 210 (44.2\%) mencionaron prescribir bifosfonatos. Las enfermedades con su frecuencia para las que los médicos entrevistados utilizan bifosfonatos se muestran en la Tabla 1.

Tabla 1: Distribución de las enfermedades para las cuales se prescribieron bifosfonatos.

\begin{tabular}{lc} 
Enfermedad & $\mathrm{n}(\%)$ \\
\hline Osteoporosis & $71(33.8)$ \\
Cáncer de próstata & $39(18.6)$ \\
Osteopenia & $31(14.8)$ \\
Cáncer óseo & $22(10.5)$ \\
Otros tipos de cáncer & $22(10.5)$ \\
Cáncer de mama & $13(6.2)$ \\
Mieloma múltiple & $7(3.3)$ \\
Hipercalcemia & $4(1.9)$ \\
Enfermedad de Paget & $1(0.4)$ \\
\hline
\end{tabular}

Respecto a la prevención de osteonecrosis de los maxilares por bifosfonatos, $58.1 \%$ de los médicos encuestados que prescriben estos medicamentos no envían a los pacientes con el odontólogo para eliminar factores de riesgo, a pesar de que $61.8 \%$ de los mismos mencionó conocer las reacciones adversas en los tejidos bucales. Específicamente sobre la osteonecrosis por bifosfonatos, sólo $29 \%$ de los médicos que prescriben bifosfonatos se ha actualizado respecto a esta patología; 55\% mencionó estar familiarizado con el término; y 36 médicos (17.4\%) han visto a algún paciente con OMRB. Respecto al diagnóstico de esta entidad, 37\% (76 entrevistados) de los médicos que prescriben medicamentos consideran que no es necesario remitir a los pacientes que recibirán o ya están bajo tratamiento con estos medicamentos. El 64.7\% no conocen los signos y síntomas de la OMRB, 21.7\% sólo conocen un signo o síntoma de este padecimiento y sólo 13.5\% conocen dos o más características clínicas que determinan el diagnóstico de la OMRB. Con respecto a los factores de riesgo para el desarrollo de OMRB, 63.3\% no los conoce, $22.7 \%$ sólo conoce uno y $14 \%$ conoce dos o más factores de riesgo.

Al analizar la especialidad de los médicos entrevistados con sus conocimientos respecto al diagnóstico y prevención, los ginecólogos, urólogos, cirujanos y los médicos familiares conocen más sobre los nombres comerciales y sustancia activa de los bifosfonatos.

Los especialistas que con más frecuencia remiten a sus pacientes bajo tratamiento con bifosfonatos al odontólogo son: oncólogos, ginecólogos, pediatras y cirujanos ( $p=0.001$; los médicos familiares y los médicos internistas son los clínicos que conocen más sobre los signos y síntomas $(p=0.000)$; sobre los factores de riesgo los médicos familiares y los oncólogos son los médicos que los identifican mejor $(p=0.000)$.

\section{DISCUSIÓN}

Para que un paciente desarrolle OMRB es casi indispensable que un médico haya prescrito dichos medicamentos, ya que estos fármacos son aún poco conocidos por el público en general y no se autorrecetan, por lo tanto, se podría decir que el médico es el primer eslabón en la cadena que lleva a esta complicación. En el presente estudio $61.5 \%$ de los entrevistados reportó tener conocimiento sobre los medicamentos y 44.2\% de los médicos los están prescribiendo activamente; sin embargo, el dato que resulta más preocupante es el hecho de que $58.1 \%$ de aquellos médicos que prescriben bifosfonatos no envían a sus pacientes al odontólogo para valoración 
previo a iniciar el tratamiento y aún más alarmante es el hecho de que $37 \%$ de los médicos que prescriben bifosfonatos considera que no es necesario referirlos al odontólogo. Esta situación, aunada al poco conocimiento de los odontólogos sobre el tema, ${ }^{13}$ está generando que los casos de osteonecrosis relacionadas con bifosfonatos vayan en aumento. En $2011 \mathrm{Al}$-Mohaya y colaboradores publicaron un estudio realizado en Arabia Saudita en el cual encuestaron a médicos y odontólogos, encontraron que sólo $31.5 \%$ de los encuestados tenían conocimiento adecuado sobre el tema; ${ }^{14}$ Osta y su equipo en 2015 realizaron un estudio similar para evaluar el conocimiento sobre el tema en los médicos en Líbano, encontrando que $37.5 \%$ de los encuestados no estaban al tanto de esta complicación y en general el conocimiento de los médicos al respecto fue pobre. ${ }^{15}$ Kim y colegas en 2016 publicaron un estudio sobre la percepción de los médicos respecto a la OMRB en Corea del Sur, hallaron que 21.9\% de los encuestados no habían escuchado sobre esta complicación y menos de $30 \%$ de los médicos refieren a sus pacientes al odontólogo previo a iniciar el tratamiento con bifosfonatos. ${ }^{16}$ Estos estudios reportan resultados similares al que estamos presentando, lo cual pudiera estar relacionado con que todos se hicieron en países en vías de desarrollo, no se encontraron estudios semejantes en países desarrollados en los que pudiera existir diferencia en los resultados.

En cuanto a las diferencias por especialidad, los oncólogos se encuentran en el grupo de médicos que con mayor frecuencia refieren a los pacientes al odontólogo previo al tratamiento y están mayormente familiarizados con la identificación de esta complicación, estos resultados confirman lo publicado por Senturk y su grupo en 2016, quienes reportan que los oncólogos entrevistados tienen un conocimiento suficiente y realizan interconsultas con el odontólogo $;{ }^{17}$ sin embargo, la muestra de su estudio es pequeña, ya que únicamente entrevistaron a 53 especialistas. El hecho de que los oncólogos sean los especialistas con mayor tasa de referencia y con un mayor conocimiento de esta complicación podría estar relacionado a que el uso de los bifosfonatos en su especialidad vaya en aumento y en la mayoría de los casos por vía intravenosa en donde el riesgo de presentar osteonecrosis es mayor.

Como en muchas enfermedades la prevención juega un rol fundamental, y la OMRB no es la excepción, de hecho, se considera una reacción adversa altamente prevenible; sin embargo, para esto se requiere de un conocimiento adecuado tanto de los médicos que prescriben como de los odontólogos que atienden a este grupo de pacientes. Los bifosfonatos resultan de gran utilidad para el tratamiento o prevención de distintas condiciones médicas, por lo que no se critica el uso como tal de los medicamentos, únicamente se sugiere establecer protocolos estrictos en los que el paciente sea canalizado al odontólogo para una valoración y tratamiento previo al inicio de la terapia con estos fármacos, similar a lo que ya se realiza en el caso de la radiación en cabeza y cueIlo. Con base en los resultados obtenidos, es imperativo implementar estrategias que incrementen la conciencia global sobre esta complicación y estimulen a la prevención de la misma. Las asociaciones médicas y odontológicas deberían colaborar con la difusión entre sus agremiados; se deben implementar programas de educación continua para los médicos generales y especialistas, así como contemplarlo en los planes de estudio de las carreras de medicina y odontología. Asimismo, los laboratorios farmacéuticos deberían ser más enfáticos en instruir al personal de salud y a los pacientes sobre las posibles complicaciones de estos medicamentos.

\section{CONCLUSIONES}

La prescripción de bifosfonatos en la práctica médica va en aumento, la osteonecrosis relacionada con bifosfonatos es una complicación poco frecuente; sin embargo, cuando se presenta su tratamiento puede resultar complejo y en ocasiones debe ser agresivo, lo cual implica una morbilidad importante para el paciente. Esta complicación podría prevenirse si tanto el médico como el odontólogo llevan a cabo protocolos estrictos. El médico, ya que es quien prescribe estos medicamentos, debe tener conocimiento adecuado de las posibles complicaciones y poder referir de manera oportuna al paciente con el odontólogo. Además, es imperativo reforzar las estrategias de difusión sobre las complicaciones de estos medicamentos entre el personal de salud y los pacientes que los consumen.

\section{AGRADECIMIENTOS}

A la Dra. Martha Reyes Mercado por su valiosa ayuda en la realización de este proyecto.

\section{REFERENCIAS}

1. Gavaldá C, Bagán JV. Concept, diagnosis and classification of bisphosponate-associated osteonecrosis of the jaws. A review of the literature. Med Oral Patol Oral Cir Bucal. 2016; 2 (3): e260-e270.

2. Moro-Alvarez MJ, Díaz-Curiel M. Risedronate once monthly: a potential new regimen for the treatment of postmenopausal osteoporosis. Clin Interv Aging. 2008; 3 (2): 227-232. 
3. He Y, Huang Y, Huang Z, Jiang Y, Sun X, Shen Y et al. Bisphosphonatefunctionalized coordination polymer nanoparticles for the treatment of bone metastatic breast cancer. J Control Release. 2017; 264: 76-88.

4. Marx RE. Pamidronate (Aredia) and zoledronate (Zometa) induced avascular necrosis of the jaws: a growing epidemic. J Oral Maxillofac Surg. 2003; 61 (9): 1115-1117.

5. Ruggiero SL, Mehrotra B, Rosenberg TJ, Engroff SL. Osteonecrosis of the jaws associated with the use of bisphosphonates: a review of 63 cases. J Oral Maxillofac Surg. 2004; 62 (5): 527-534.

6. Advisory Task Force on Bisphosphonate-Related Ostenonecrosis of the Jaws, American Association of Oral and Maxillofacial Surgeons. American Association of Oral and Maxillofacial Surgeons position paper on bisphosphonate-related osteonecrosis of the jaws. J Oral Maxillofac Surg. 2007; 65 (3): 369-376.

7. Ruggiero SL, Dodson TB, Assael LA, Landesberg R, Marx RE, Mehrotra B. American Association of Oral and Maxillofacial Surgeons position paper on bisphosphonate-related osteonecrosis of the jaws--2009 update. J Oral Maxillofac Surg. 2009; 67 (5 Suppl): 2-12.

8. Ruggiero SL, Dodson TB, Fantasia J, Goodday R, Aghaloo T, Mehrotra B et al. American Association of Oral and Maxillofacial Surgeons position paper on medication-related osteonecrosis of the jaw--2014 update. J Oral Maxillofac Surg. 2014; 72 (10): 19381956.

9. Manfredi M, Mergoni G, Goldoni M, Salvagni S, Merigo E, Meleti $M$ et al. A 5-year retrospective longitudinal study on the incidence and the risk factors of osteonecrosis of the jaws in patients treated with zoledronic acid for bone metastases from solid tumors. Med Oral Patol Oral Cir Bucal. 2017; 22 (3): e342-e348.

10. Khan AA, Morrison A, Hanley DA, Felsenberg D, McCauley LK, $\mathrm{O}^{\prime}$ Ryan $\mathrm{F}$ et al. Diagnosis and management of osteonecrosis of the jaw: a systematic review and international consensus. J Bone Miner Res. 2015; 30 (1): 3-23.
11. Yoo JY, Park YD, Kwon YD, Kim DY, Ohe JY. Survey of Korean Dentist on the awareness on bisphosphonate-related osteonecrosis of the jaws. J Investig Clin Dent. 2010; 1 (2): 90-95.

12. Alhussain A, Peel S, Dempster L, Clokie C, Azarpazhooh A. Knowledge, practices, and opinions of Ontario dentists when treating patients receiving bisphosphonates. J Oral Maxillofac Surg. 2015; 73 (6): 1095-1105.

13. Vinitzky-Brener I, Ibáñez-Mancera NG, Aguilar-Rojas AM, ÁlvarezJardón AP. Knowledge of bisphosphonate-related osteonecrosis of the Jaws among Mexican dentists. Med Oral Patol Oral Cir Bucal. 2017; 22 (1): e84-e87.

14. Al-Mohaya MA, Al-Khashan HI, Mishriky AM, Al-Otaibi LM. Physicians' awareness of bisphosphonates-related osteonecrosis of the jaw. Saudi Med J. 2011; 32 (8): 830-835.

15. El Osta L, El Osta B, Lakiss S, Hennequin M, El Osta N. Bisphosphonate-related osteonecrosis of the jaw: awareness and level of knowledge of Lebanese physicians. Support Care Cancer. 2015; 23 (9): 2825-2831.

16. Kim JW, Jeong SR, Kim SJ, Kim Y. Perceptions of medical doctors on bisphosphonate-related osteonecrosis of the jaw. BMC Oral Health. 2016; 16 (1): 92.

17. Senturk MF, Cimen E, Tuzuner Oncul AM, Cambazoglu M. Oncologists awareness about bisphosphonate related osteonecrosis of the jaws. J Pak Med Assoc. 2016; 66 (7): 880-883.

\section{Correspondencia: \\ Dr. Ilan Vinitzky-Brener \\ E-mail: ilanvinitzky@hotmail.com \\ ORCID: 0000-0001-8378-1444.}

Conflicto de intereses: Los autores declaran no tener ningún conflicto de intereses. 